\title{
Galactic members in the New Online Database of Symbiotic Variables
}

\author{
J. Merc ${ }^{1,2}$, R. Gális ${ }^{1}$ and M. Wolf ${ }^{2}$ \\ 1 Institute of Physics, Faculty of Science, P. J. Šafárik University, Park \\ Angelinum 9, 04001 Košice, Slovak Republic \\ 2 Astronomical Institute, Faculty of Mathematics and Physics, Charles \\ University, V Holešovičkách 2, 18000 Prague, Czech Republic
}

Received: October 23, 2019; Accepted: December 2, 2019

\begin{abstract}
Symbiotic variables belong to an interesting class of interacting binary stars. At the beginning of this century, a systematic search for these objects was begun and such surveys in the Milky Way and the Local Group have resulted in discoveries of many new symbiotics and dozens of candidates. Because the latest catalogue of symbiotic binaries has been outdated for almost two decades, we decided to prepare a new database of the Galactic and extra-Galactic symbiotic systems. We present it in this work. Our database is available online, allowing it to be up-to-date and available to the astronomical community at any time. The database also includes a web portal that allows easy data access without the necessity for any additional software or formatting of the data.
\end{abstract}

Key words: catalogues — binaries: symbiotic

\section{Symbiotic stars}

Symbiotic variables are the widest interacting binaries consisting of a cool giant (or a supergiant) of a spectral type $\mathrm{K}$ or $\mathrm{M}$ (rarely G) as the donor and a compact star, most commonly a hot (about $10^{5} \mathrm{~K}$ ) white dwarf ${ }^{1}$ as the accretor of transferred matter (Mikołajewska, 2007).

Mass transfer between the components most likely takes place via the cool giant's wind (e.g. Mikołajewska, 2007), which is also the source of a dense circumbinary environment. The spectra of these objects are therefore a superposition of three components of radiation - two stellar and one nebular (e.g. Skopal, 2005). The cool giant usually dominates the spectrum at longer wavelengths (in the IR) and the hot component mainly radiates in the UV and blue part of the optical region. Symbiotic spectra are often rich in emission lines (Balmer lines of $\mathrm{H}$, and neutral and ionized $\mathrm{He}, \mathrm{Fe}$ lines). Emission lines of a special interest are Raman-scattered lines of $\mathrm{O}$ VI which are an exclusive feature of symbiotic

\footnotetext{
${ }^{1}$ It is worth noting that several symbiotic binaries with accreting neutron stars have also been identified (e.g. Corbet et al., 2008; Enoto et al., 2014, and references therein).
} 


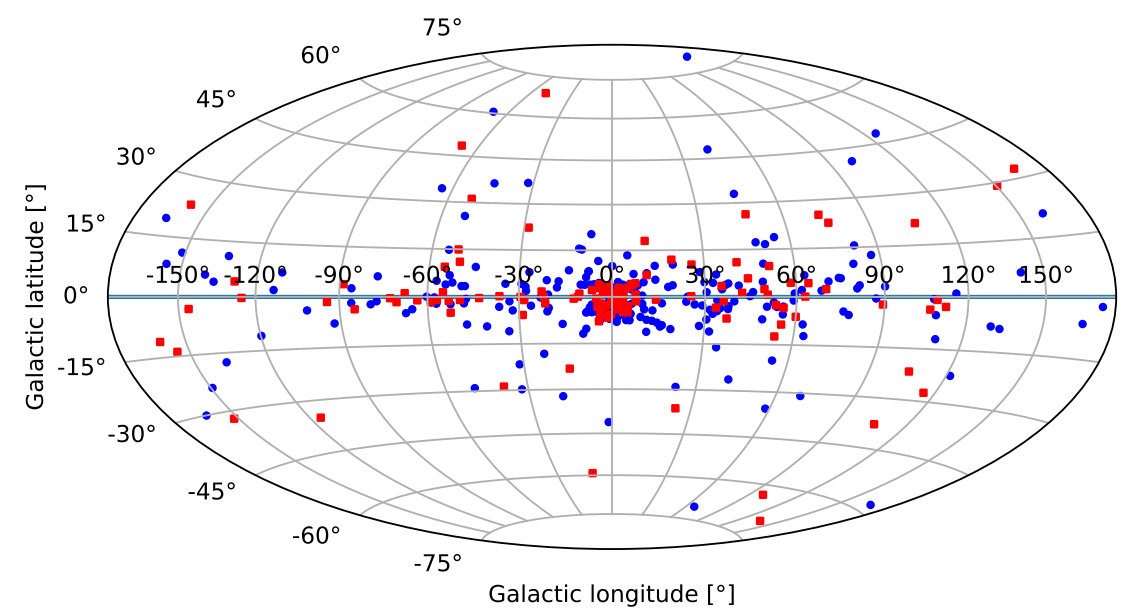

Figure 1. Distribution of the Galactic symbiotic stars according their Galactic coordinates. Confirmed and suspected symbiotic stars are denoted by blue dots and red squares, respectively.

binaries (Akras et al., 2019) and provide a strong criterion for spectroscopic identification of new symbiotics.

Since the beginning of the 21st century, a systematic search for symbiotic binaries has been running, and not only in the Milky Way (e.g. Miszalski et al., 2013; Miszalski \& Mikołajewska, 2014) but also beyond it in nearby galaxies. Some surveys have resulted in the identification of new extra-Galactic symbiotic variables or at least promising candidates (e.g. Gonçalves et al., 2008, 2015; Kniazev et al., 2009; Mikołajewska et al., 2014, 2017). Thanks to these surveys, the number of known symbiotic systems is growing rapidly.

\section{New Online Database of Symbiotic Variables}

Based on the recent progress in the search for and study of symbiotic stars, we have decided to prepare a new database of these interacting binaries. The purpose of the database is not only to serve as a catalogue of data for all known symbiotics with consistent references, but we have also prepared a web-portal for easy access to this information. Making the database online allows us to add new objects as soon as they are discovered and update or complete data when new information becomes available. In this way, up-to-date lists of symbiotic variables and information about particular objects could be available to the astronomical community at any time.

The full version of the database contains all available data on the location of objects, their brightness in various spectral regions and other observational 
properties (such as the presence of outbursts, flickering, detectable X-ray or radio emission and symbiotic type). The database also provides information on orbital elements (periods, ephemerides, presence of eclipses etc.) and parameters of the binary components (spectral types, temperatures, masses, radii, luminosities, presence of pulsations etc.).

For the catalogued symbiotic stars, we have prepared specific object pages covering all available information included in the database. The data provided are accompanied by appropriate references that are directly linked to quoted articles in the ADS database, making it easier and faster to find them. The latest version of the New Online Database of Symbiotic Variables is available at the internet address http://astronomy.science.upjs.sk/symbiotics/.

The extra-galactic part of the database was presented by (Merc et al., 2019). We are continuing to prepare the Galactic part of the database. The list of all confirmed and suspected objects with their basic parameters is already available. The distribution of the Galactic symbiotic stars according their galactic coordinates is shown in Fig. 1.

Almost all Galactic symbiotic stars are located around the Milky Way equator $\left(|b|<15^{\circ}\right)$. For the objects in the current version of the database, $89 \%$ of the confirmed and $83 \%$ of candidate symbiotics are located in this sky region. The distribution in Galactic longitude demonstrates that symbiotics are located mostly towards the galactic bulge (56\% and $52 \%$ of the confirmed and candidate symbiotics within $|l|<30^{\circ}$, respectively). However these ratios are biased by a selective effect because surveys tend to focus on the surroundings of the Galactic equator and also the density of stars is higher in the Galactic disk.

\section{Conclusions}

Symbiotic binaries can serve as unique astrophysical laboratories in the study of accretion processes, winds and jets. Moreover, they are important for evolutionary models as they may be one of the type Ia supernova progenitors. However, a proper characterization of the symbiotic population is still lacking. The systematic search for these objects, which has begun recently, has caused the number of known systems to grow rapidly. This has led to the need for a new catalogue of symbiotic binaries that allows systematic studies. For this reason we are preparing the New Online Database of Symbiotic Variables, which would serve not only as a catalogue of all available information on particular symbiotic stars but also as a web portal for easy access to these data. The extra-Galactic part of the database is already filled and an updated list of Galactic symbiotic stars has been recently released.

Acknowledgements. This research was supported by the Slovak Research and Development Agency grant No. APVV-15-0458 and by the Faculty of Science, P. J. Šafárik University in Košice under the internal grant VVGS-PF-2019-1047. 


\section{References}

Akras, S., Guzman-Ramirez, L., Leal-Ferreira, M. L., \& Ramos-Larios, G., A Census of Symbiotic Stars in the 2MASS, WISE, and Gaia Surveys. 2019, Astrophys. J., Suppl., 240, 21, DOI: 10.3847/1538-4365/aaf88c

Corbet, R. H. D., Sokoloski, J. L., Mukai, K., Markwardt, C. B., \& Tueller, J., A Comparison of the Variability of the Symbiotic X-Ray Binaries GX 1+4, 4U 1954+31, and $4 \mathrm{U} 1700+24$ from Swift BAT and RXTE ASM Observations. 2008, Astrophys. J., 675, 1424, DOI: 10.1086/526337

Enoto, T., Sasano, M., Yamada, S., et al., Spectral and Timing Nature of the Symbiotic X-Ray Binary 4U 1954+319: The Slowest Rotating Neutron Star in an X-Ray Binary System. 2014, Astrophys. J., 786, 127, DOI: 10.1088/0004-637X/786/2/127

Gonçalves, D. R., Magrini, L., de la Rosa, I. G., \& Akras, S., Discovery of true, likely and possible symbiotic stars in the dwarf spheroidal NGC 205. 2015, Mon. Not. R. Astron. Soc., 447, 993, DOI: 10.1093/mnras/stu2437

Gonçalves, D. R., Magrini, L., Munari, U., Corradi, R. L. M., \& Costa, R. D. D., Discovery in IC10 of the farthest known symbiotic star. 2008, Mon. Not. R. Astron. Soc., 391, L84, DOI: 10.1111/j.1745-3933.2008.00561.x

Kniazev, A. Y., Väisänen, P., Whitelock, P. A., et al., Discovery of the first symbiotic star in NGC6822. 2009, Mon. Not. R. Astron. Soc., 395, 1121, DOI: 10.1111/j.13652966.2009.14617.x

Merc, J., Gális, R., \& Wolf, M., First Release of the New Online Database of Symbiotic Variables. 2019, Research Notes of the American Astronomical Society, 3, 28, DOI: $10.3847 / 2515-5172 /$ ab0429

Mikołajewska, J., Symbiotic Stars: Continually Embarrassing Binaries. 2007, Baltic Astronomy, 16, 1

Mikołajewska, J., Caldwell, N., \& Shara, M. M., First detection and characterization of symbiotic stars in M31. 2014, Mon. Not. R. Astron. Soc., 444, 586, DOI: $10.1093 / \mathrm{mnras} / \mathrm{stu} 1480$

Mikołajewska, J., Shara, M. M., Caldwell, N., Iłkiewicz, K., \& Zurek, D., A survey of the Local Group of galaxies for symbiotic binary stars - I. First detection of symbiotic stars in M33. 2017, Mon. Not. R. Astron. Soc., 465, 1699, DOI: 10.1093/mn$\mathrm{ras} / \mathrm{stw} 2937$

Miszalski, B. \& Mikołajewska, J., Identification of new Galactic symbiotic stars with SALT - I. Initial discoveries and other emission line objects. 2014, Mon. Not. R. Astron. Soc., 440, 1410, DOI: 10.1093/mnras/stu292

Miszalski, B., Mikołajewska, J., \& Udalski, A., Symbiotic stars and other H $\alpha$ emissionline stars towards the Galactic bulge. 2013, Mon. Not. R. Astron. Soc., 432, 3186, DOI: $10.1093 / \mathrm{mnras} / \mathrm{stt} 673$

Skopal, A., Disentangling the composite continuum of symbiotic binaries. I. S-type systems. 2005, Astron. Astrophys., 440, 995, DOI: 10.1051/0004-6361:20034262 